\section{Metabolic changes in malnutrition}

PW Emery

Eye (2005) 19, 1029-1034. doi:10.1038/sj.eye.6701959

Keywords: malnutrition; protein metabolism; energy metabolism; adaptation; wound healing

\section{Malnutrition}

This paper will be concerned mainly with what Golden refers to as type II nutrients (Table $1^{1}$ ). I have added energy, fat, and carbohydrate to the original list even though they do not meet the strict definition of essential nutrients. The type II nutrients are found as components of all tissues, in relatively fixed amounts. A lack of any one of these type II nutrients causes an immediate cessation of growth and an inability to maintain the balance of the other type II nutrients. Hence the signs of such a deficiency are relatively nonspecific, because they could all be attributable to a lack of any of these nutrients. Moreover, it does not matter which of these nutrients is the most deficient, because in most naturally occurring situations the intake of all of them is likely to be deficient, and there will be no restoration of function until there is adequate intake of all of them.

Malnutrition may arise acutely or chronically, and the metabolic response may be slightly different in each case. It may be helpful to consider three general situations. The first is young children in third world countries suffering from varying degrees of proteinenergy malnutrition. Young children are uniquely vulnerable to the effects of inadequate food intake and chronic exposure to infectious illnesses, resulting in high death rates.

Malnutrition may not always be recorded as the immediate cause of death but it has been estimated to account for $35 \%$ of the deaths of children worldwide. ${ }^{2}$ The second situation is the large numbers of older children and adults, again mainly in third world countries, who have survived childhood but who are living on marginally inadequate intakes, and are typically classified as chronically energy deficient. Although such people are considered to have
Department of Nutrition and Dietetics, King's College London, London, UK

Correspondence: PW Emery، Department of Nutrition and Dietetics, King's College London, Franklin Wilkins Building, 150 Stamford Street, London SE1 9NH, UK Tel: + 44207848 4415; Fax: +44207848 4185 . E-mail: peter.emery@ kcl.ac.uk

Received: 16 September 2004

Accepted: 2 May 2005 
Table 1 Type I and Type II nutrients

Type I
All vitamins, most trace elements, calcium
Type II
Nitrogen, sulphur, essential amino acids
Potassium, sodium, magnesium, phosphorus, zinc
Water
Dietary sources of energy (including carbohydrate and fat)

Adapted from Golden and Golden. ${ }^{1}$

adapted successfully to their inadequate diet their work output is low and their ability to perform and enjoy normal social functions may be limited. The third situation occurs more commonly in industrialised countries and relates to people with severe illnesses that lead to anorexia and wasting. The resulting malnutrition becomes a synergistic factor contributing to further morbidity and mortality.

In all these cases the primary cause of malnutrition is inadequate food intake, but in most cases there is also an effect of disease which causes an increase in requirement for protein and energy. The disease may be an infection, such as diarrhoea or measles in the malnourished child, or it may be a chronic inflammatory process or an acute trauma such as a hip fracture or surgery in the case malnourished adults in hospitals. Such disease processes also exacerbate the malnutrition by causing anorexia. Malnutrition in turn exacerbates the disease process by suppressing immune function. The interaction between malnutrition and disease, particularly infectious disease, is often described as a vicious circle. ${ }^{3}$

The metabolic changes that are observed in these situations are difficult to interpret since they represent the response to both malnutrition and disease. Some data on the effects of pure undernutrition in human subjects comes from the Minnesota experiment ${ }^{4}$ and from some more recent studies of obese people who are dieting to lose weight. Experimental studies with human volunteers tend to focus mainly on relatively short periods of complete starvation. However, much useful information can also be gained from studies in animal models. We have used this approach to study the interaction between malnutrition and the metabolic response to injury (see below).

\section{Metabolic changes in young children with protein-energy malnutrition}

The immediate effect of inadequate intake in a child will be for growth to slow or stop, so that protein and energy can be used for maintenance of the existing tissue. If the energy deficit is so severe that energy balance cannot be maintained there will be a loss of body tissue, both fat and lean, to provide substrates to maintain the metabolism of the remaining cells. Thus chronic malnutrition results in slow growth, and can be detected by measuring height (or length, in the case of infants) for age, with low values being classified as stunting. More acute malnutrition will tend to cause a decrease in weight for height, and this is classified as wasting.

In extreme cases malnutrition leads to the syndromes known as marasmus and kwashiorkor. It has been suggested that marasmus represents the extreme result of adaptation to low intake, with complete cessation of growth and severe loss of muscle and adipose tissue, while the features of kwashiorkor such as oedema and fatty liver may represent a failure in some aspect of this adaptation. However, the biochemical changes that have been reported are not consistent enough to give many reliable clues as to the nature of any metabolic differences. Certainly, the old notion that kwashiorkor was caused by a diet that was uniquely low in protein and that this caused a failure to secrete albumin and apolipoproteins is not supported by the evidence.

In fact, the diets of children with kwashiorkor are often not particularly low in the ratio of protein to energy, but rather they are too dilute so that the intake of both protein and energy is low. ${ }^{5,6}$ Moreover, children can recover effectively on diets that would be considered low in protein. ${ }^{7}$ The plasma protein concentration is often not particularly low in children with oedema, and during rehabilitation the oedema may clear without any change in serum albumin concentration. ${ }^{7}$

One theory for which there is a growing amount of evidence invokes the role of free radicals in causing tissue damage, including disrupting export protein synthesis in the liver and causing leakage of fluid from the capillaries. Free radical damage may be triggered by environmental or food-borne toxins or infections acting on cells with excessive levels of free iron and inadequate amounts of protective molecules such as reduced glutathione, which in turn may be a result of inadequate intake of antioxidant nutrients. ${ }^{7}$ Free radical damage to the cell membrane causes intracellular concentrations of sodium to rise and potassium to fall.

\section{Metabolic response to starvation}

The immediate response to the initiation of starvation is similar to the normal changes that occur in the postabsorptive phase between meals. Most tissue utilise glucose as a fuel immediately after a meal, then switch progressively to the use of fatty acids. However, the brain has an obligatory requirement for glucose, as do the lens of the eye, the red blood cells, the skin, and the kidney 
medulla. Hence, the circulating concentration of glucose must be maintained by the breakdown of glycogen that is stored in the liver after each meal. Once glycogen supplies are used up glucose must be produced entirely by gluconeogenesis, initially mainly in the liver but the kidney plays an increasing role as starvation progresses. The main substrates are amino acids, particularly alanine and glutamine, together with glycerol, which is produced when stored triglycerides are broken down, and lactate, which is produced by glycolysis. As fasting continues the liver also begins to produce ketone bodies, principally 3-hydroxybutyrate and some acetoacetate, which can be used by the brain and this reduces the amount of glucose that must be produced.

Thus with prolonged fasting the body adapts to use its major energy reserve, the triglyceride that is stored in adipose tissue. The rate at which lean tissue is broken down to supply amino acids for gluconeogenesis is minimised, because of the functional consequences of loss of proteins, so the amount of nitrogen excreted in the urine decreases as fasting progresses. Thus the amount of glucose used by the brain decreases from around $80 \mathrm{~g} /$ day in the early stages to approximately $35 \mathrm{~g} /$ day in long-term starvation, with the rest of the energy requirement being met from ketones. ${ }^{8}$

It has generally been assumed that skeletal muscle protein is the main source of amino acids during starvation. However, we have shown in fasting rats that the smooth muscle of the gut breaks down rather more rapidly than skeletal muscle during the initial stages of starvation. ${ }^{9}$ This may have functional consequences both for the function of the gut as a barrier to bacterial translocation and for the ability to absorb nutrients when refeeding begins. The amount of protein present as smooth muscle is also relatively small in comparison to the amount of skeletal muscle, so skeletal muscle soon becomes the main source of amino acids. Fasting appears to affect all skeletal muscles equally, ${ }^{10}$ although there may be a greater loss of type II fibres than type I fibres. ${ }^{11}$ Muscle function is impaired, with some evidence that muscle fatiguability is disproportionately increased in malnutrition. ${ }^{12}$ The most serious consequences are associated with the loss of respiratory muscle leading to impaired ability to cough and thus increased susceptibility to respiratory infections.

The basal metabolic rate actually increases during the first few days of starvation, under the influence of catecholamines that are secreted in response to decreasing blood glucose concentrations. ${ }^{13,14}$ This probably reflects the high rate of gluconeogenesis that occurs at this time. As fasting progresses, however, metabolic rate decreases as free $\mathrm{T}_{3}$ and catecholamine levels decrease and the rate of gluconeogenesis decreases.

\section{Metabolic response to underfeeding}

The response to a less severe degree of food restriction can also be seen as a series of adaptive processes with the same priorities, that is to maintain the supply of glucose to the brain and to minimise the loss of lean tissue. Basal metabolic rate decreases to minimise the negative energy balance. This is achieved partly by loss of metabolically active tissue, but there is also some evidence that the efficiency of energy metabolism increases leading to a decrease in energy expenditure per unit cell mass. ${ }^{15}$ However, it is not clear what the mechanism for this improved efficiency is. There may be some scope for increasing the efficiency of coupling of ATP production to substrate oxidation by preventing mitochondrial proton leakage. There may also be some reduction in protein turnover and the activity of sodium/potassium ATPase, which are the processes that account for the majority of basal energy expenditure.

In the longer term, however, the main mechanism by which energy expenditure is minimised is by the progressive loss of metabolically active tissue. Thus, for example, in the Minnesota experiment ${ }^{4}$ after 168 days of 'semistarvation' metabolic rate had decreased by $37 \%$ when expressed as kcal/day but by only $15 \%$ when expressed as $\mathrm{kcal} /$ day per unit active tissue mass.

\section{Adaptation to chronic energy restriction}

It appears that the adaptation of energy metabolism in people who live their lives on marginally inadequate food intakes may be somewhat different from the response of previously well-nourished subjects undergoing a period of underfeeding. The main response in chronically malnourished populations is slow growth rate, delayed maturity, and small adult stature. Small stature can be seen as a successful adaptation to lowenergy intake because overall basal metabolic rate will be low. However, when metabolic rate is adjusted for fatfree mass there is no significant difference between those who are most malnourished and those who are well nourished. ${ }^{16}$ The reason for this is that the main deficit in lean tissue mass is in muscle, which has a relatively low metabolic rate, while the size of the visceral organs, which are much more metabolically active, is much less affected. ${ }^{17}$ Hence these changes in body composition may cancel out any increase in the efficiency of cellular metabolism.

There are also reductions in other components of total energy expenditure. There may be reductions in both cold- and diet-induced thermogenesis, though the evidence is not conclusive. ${ }^{15}$ On the other hand, there is consistent evidence of a reduction in the energy expenditure associated with physical activity in undernourished people. Part of this comes again from 
the reduction in body size. There are also behavioural changes, with work being done more slowly and more efficiently, and with leisure time activities being more likely to be sedentary. ${ }^{15}$ Energy expenditure after exercise may also return to normal more rapidly. ${ }^{18}$

\section{Effect of malnutrition on the metabolic response to trauma}

The metabolic changes that follow acute trauma are somewhat different from those that accompany chronic malnutrition. The work of Cuthbertson ${ }^{19}$ showed that trauma in the form of either accidental injury or surgery causes an extended period of increased energy expenditure and increased nitrogen excretion in the urine. $^{20}$ These responses reflect increased gluconeogenesis, lipolysis, and net breakdown of muscle protein in order to mobilise substrates and energy to support the activation of inflammatory and reparative processes to promote recovery. Since people who undergo surgery are often somewhat malnourished as a result of a chronic disease process, it is important to know whether malnourished patients are able to mount the same metabolic response to trauma.

We investigated this question in a group of female rats that were malnourished by feeding them on a $2 \%$ protein diet and restricting their intake to $50 \%$ of the intake of a control group, which was fed ad libitum on a $17 \%$ protein diet. After 3 weeks half the rats underwent hysterectomy and were then returned to the same dietary treatment for a further 4 days while the other rats were kept on the same dietary treatment but pair-fed with the operated rats. At the end of the experiment all the rats were killed, the carcasses were analysed for protein and fat content and energy balance was calculated. The ad libitum fed rats showed a $37 \%$ increase in energy expenditure after surgery, as expected. The malnourished rats also showed an increase in energy expenditure, but it was considerably smaller, at $22 \%$ of the control value. (Table $2^{21}$ ). In a separate experiment we measured urinary nitrogen excretion after surgery in similar groups of rats. In the well-nourished rats there was a considerable increase in urinary nitrogen excretion on each of the first three days after surgery, but in the malnourished rats there was a small rise only on the second day (Table $2^{21}$ ). Hence, it appears that malnourished animals are able to mount a metabolic response to surgery, but its magnitude is considerably smaller than that in well-nourished animals. This may contribute to the increased vulnerability of malnourished patients to complications following surgery.

\section{The effect of malnutrition on wound healing}

It is commonly believed that malnutrition has a deleterious effect on the wound healing process, and that this represents an important mechanism by which malnourished patients are liable to develop complications and have poorer outcome after surgery. This view is derived largely from clinical observations rather than from any reliable experimental data in man. ${ }^{22}$ Studies in experimental animals suggest that wound healing is only impaired after prolonged dietary restriction resulting in severe depletion. ${ }^{23}$ We have investigated the metabolic basis of the relationship between nutritional status and wound healing by measuring changes in the rate of protein synthesis within the healing wound. We had previously shown that the rate of protein synthesis more than doubles during wound healing in muscle, and we hypothesised that this may be one of the mechanisms by which nutritional status affects would healing, since malnutrition is known to reduce the rate of protein synthesis and might thus prevent the deposition of new protein to heal the wound. ${ }^{24}$

Our studies have focused on the healing of abdominal muscle after abdominal surgery. In the first experiment, rats were malnourished by restricting their food intake to $50 \%$ of normal for 7 days. This resulted in a $14 \%$ deficit in body weight. They were then subjected to a laparotomy and maintained on the restricted diet for a further 2 days, after which protein synthesis was measured in vivo using the flooding dose technique. ${ }^{25}$ Although this dietary treatment caused a significant reduction in the rate of

Table 2 Energy balance and urinary nitrogen excretion following surgery in malnourished and ad lib fed control rats

\begin{tabular}{|c|c|c|c|c|}
\hline & \multicolumn{2}{|c|}{ Ad lib fed rats } & \multicolumn{2}{|c|}{ Malnourished rats } \\
\hline & Surgical & Control & Surgical & Control \\
\hline Energy intake (kJ/4 days) & $623(29)$ & $614(33)$ & 278 & 278 \\
\hline Energy expenditure (kJ/4 days) & $874(42)^{*}$ & $640(33)$ & $355(8)^{*}$ & $291(15)$ \\
\hline Urine nitrogen ( $\mathrm{g} / 4$ days) & $1101(83)^{*}$ & $685(43)$ & $258(21)^{*}$ & $198(19)$ \\
\hline
\end{tabular}

Values are mean (SE) for groups of 6-8 rats.

*Significantly different from corresponding control group, $P<0.05$

Data taken from Emery et al. ${ }^{21}$ 
Table 3 Protein synthesis rate in muscle at the wound site, uninjured abdominal muscle, and gastrocnemius muscle $48 \mathrm{~h}$ after surgery in malnourished and ad lib fed control rats

\begin{tabular}{|c|c|c|c|c|c|c|c|}
\hline \multirow[t]{2}{*}{ Protein synthesis (\%/day) } & \multicolumn{2}{|c|}{ Ad lib fed rats } & \multicolumn{2}{|c|}{ Malnourished rats } & \multicolumn{3}{|c|}{ Analysis of Variance } \\
\hline & Control & Surgery & Control & Surgery & Surgery & Diet & Interaction \\
\hline Wound & $8.2(0.4)$ & $18.0(0.8)$ & $5.4(0.3)$ & $17.9(0.6)$ & $* * *$ & * & * \\
\hline Abdominal & $6.5(0.2)$ & $6.2(0.9)$ & $5.2(0.4)$ & $5.3(0.2)$ & NS & * & NS \\
\hline Gastrocnemius & $7.2(0.4)$ & $5.7(0.5)$ & $5.5(0.1)$ & $5.0(0.2)$ & * & $* *$ & NS \\
\hline
\end{tabular}

Values are mean (SE) for groups of $6-8$ rats.

${ }^{*} P<0.05 ;{ }^{* *} P<0.01 ;{ }^{* * *} P<0.001$.

Data taken from Emery and Sanderson. ${ }^{22}$

protein synthesis in other muscles, it did not prevent the increase in protein synthesis in the wounded muscle (Table $3^{22}$ ). We repeated the experiment with another group of malnourished rats and found that protein synthesis in the healing abdominal muscle wound was still unimpaired seven days after surgery. These rats also showed no impairment of collagen deposition or tensile strength in the wound. ${ }^{22}$

We have subsequently expanded these studies by imposing more severe degrees of undernutrition on the rats, for example, feeding a low (2\%) protein diet for 2 weeks before surgery, or starving the rats for $48 \mathrm{~h}$ after surgery (PW Emery, V Costarelli and SL Koay, unpublished data). In both cases the increase in protein synthesis in the healing wound was unimpaired.

These results may be interpreted as indicating that wound healing has a high biological priority, and is preserved even when other functions are affected by malnutrition. The same appears to apply to reproductive activity, as malnourished women are able to give birth to healthy and well-grown babies and to produce adequate quantities of breast milk. ${ }^{26}$ However, there is a contrast with the effects on malnutrition on the immune system, where malnourished people do appear to be much more susceptible to the deleterious effects of infectious diseases.

\section{References}

1 Golden MHN, Golden BE. Severe malnutrition. In: Garrow JS, James WPT, Ralph A (eds). Human Nutrition and Dietetics, 10th edn. Churchill Livingstone: Edinburgh, 2000, pp 515-526.

2 Black R, Morris S, Bryce J. Where and why are 10 million children dying every year? Lancet 2003; 361: 2226-2234.

3 Keusch GT. The history of nutrition: malnutrition, infection and immunity. J Nutr 2003; 133: 336S-340S.

4 Keys A, Brozek J, Henschel A, Mickelson O, Taylor HL. The biology of human starvation. University of Minnesota Press: Minneapolis, 1950.
5 Gopalan C. Kwashiorkor and marasmus: evolution and distinguishing features. In: McCance RA, Widdowson EM (eds). Calorie Deficiencies and Protein Deficiencies. J \& A Churchill: London, 1968, pp 48-58.

6 Naismith DJ. Kwashiorkor in western Nigeria: a study of traditional weaning foods, with particular reference to energy and essential fatty acids. Br J Nutr 1973; 30: 567-576.

7 Golden MHN. The development of concepts of malnutrition. J Nutr 2002; 132: 2117S-2122S.

8 Newsholme EA, Leech AR. Biochemistry for the Medical Sciences. John Wiley and sons: Chichester, 1983.

9 Emery PW, Cotelessa L, Holness M, Egan C, Rennie MJ. Different patterns of protein turnover in skeletal and gastrointestinal smooth muscle and the production of $\mathrm{N} \tau$-methylhistidine during fasting in the rat. Biosci Rep 1986; 6: 143-153.

10 Spence CA, Hansen-Smith FM. Comparison of the chemical and biochemical composition of thirteen muscles in the rat during protein restriction. Br J Nutr 1978; 39: 647-653.

11 Jefferson LS, Boyd TA, Flaim KE, Peavy DE. Regulation of protein synthesis in perfused preparations of rat heart, skeletal muscle and liver. Biochem Soc Trans 1980; 8: 282-283.

12 Lopes J, Russell DM, Whitwell J, Jeejeebhoy KN. Skeletal muscle function in malnutrition. Am J Clin Nutr 1982; 36: 602-610.

13 Mansell PI, Fellows IW, Macdonald IA. Enhanced thermogenic response to epinephrine after 48 -h starvation in humans. Am J Physiol 1990; 258: R87-R93.

14 Zauner C, Schneeweiss B, Kranz A, Madl C, Ratheiser K, Kramer L et al. Resting energy expenditure in short-term starvation is increased as a result of an increase in serum norepinephrine. Am J Clin Nutr 2000; 71: 1511-1515.

15 Shetty PS. Adaptation to low energy intakes: the responses and limits to low intakes in infants, children and adults. Eur J Clin Nutr 1999; 53: S14-S33.

16 Ferro-Luzzi A, Petracchi C, Kuriyan R, Kurpad AV. Basal metabolism of weight-stable chronically undernourished men and women: lack of metabolic adaptation and ethnic differences. Am J Clin Nutr 1997; 66: 1086-1093.

17 Soares MJ, Piers LS, Shetty PS, Jackson AA, Waterlow JC. Whole body protein turnover in chronically undernourished individuals. Clin Sci 1994; 86: 441-446.

18 Kulkarni RN, Kurpad AV, Shetty PS. Reduced postexercise recovery oxygen consumptions: an adaptive response in chronic energy deficiency? Metabolism 1993; 42: 544-547.

19 Cuthbertson DP. Observations on the disturbance of metabolism produced by injury to the limbs. Q J Med 1932; 25: 233-238.

20 Tilstone W, Cuthbertson DP. The protein component of the disturbance of energy metabolism in trauma. 
In: Porter R, Knight J (eds). Ciba Foundation Symposium on Energy Metabolism in Trauma. Churchill: London, 1970, pp 43-48.

21 Emery PW, Bosagh Zadeh AR, Wasylyk A. The effect of malnutrition on the metabolic response to surgery. $\mathrm{Br} \mathrm{J} \mathrm{Nutr}$ 1999; 81: 115-120.

22 Emery PW, Sanderson P. The effects of dietary restriction on protein synthesis and wound healing after surgery in the rat. Clin Sci 1995; 89: 383-388.

23 Irvin TT. Effects of malnutrition and hyperalimentation on wound healing. Surg Gynecol Obstet 1978; 146: 33-37.
24 Waterlow JC, Garlick PJ, Millward DJ. Protein Turnover in Mammalian Tissues and in the Whole Body. North-Holland: Amsterdam, 1978.

25 Garlick PJ, McNurlan MA, Preedy VR. A rapid and convenient technique for measuring the rate of protein synthesis in tissues by injection of ${ }^{3} \mathrm{H}$-phenylalanine. Biochem J 1980; 192: 719-723.

26 Naismith DJ. Protein metabolism during pregnancy. In: Philipp EE, Barnes J, Newton M (eds). Scientific Foundations of Obstetrics and Gynaecology. Heinemann: London, 1977, pp 503-510. 